\title{
Research Progress on Development and Utilization of Blueberry Wine and its Pomace
}

\author{
Songyun $\mathrm{Yu}^{*}$ \\ Wuhan Business University, College of Culinary and Food Technology, 430056 Wuhan, China
}

\begin{abstract}
Blueberry, a nutritious fruit with unique flavor, is widely used for fruit wine brewing in China. However, technical problems and the lack of diversity in nutrition and taste are factors preventing further development and utilization of blueberry for greater added value. In this paper, recent studies on blueberry compound wine and fermentation technology of blueberry beer were analyzed and summarized along with proposals on the development and utilization of blueberry fruit residue in the food manufacturing industry, serving as a reference for the development and utilization of blueberry wine and the industrial processing of blueberry beer residue.
\end{abstract}

\section{Introduction}

Sweet and sour in taste, blueberry is a fruit of sticky pectin and light fragrance, and rich in antioxidant substances such as flavonoids, anthocyanins, etc, bearing healthy implications [1]. Compared with other aquatic vegetable, blueberry is of great value as a health-care food and medicine as it carries an abundant amount of anthocyanins, earning the reputation of " healthy food" and "fruit of tremendous nutritional value" [2-5].

Blueberry is mainly used for raw food. In China, blueberry is mainly processed into fruit wine, fruit juice drinks, dairy products, candy, dried fruit and baked food [6], with fruit wine brewing taking the largest proportion of its final products. However, technical it is confronted with problems like processing techniques and the lack of diversity in nutrition and taste. So far, blueberry fruit wine has been actively developed and greatly improved, with the added value of berry wine as the key to its development and utilization [7].

The high content of water makes blueberry hard to store Blueberry features a high yield, demanding improvement of its utilization

\section{Blueberry fermented wine}

Fruit wine is a kind of low alcohol drink with $7 \%-18 \%$ alcohol concentration, which is made from fresh fruit or fruit juice and fermented in whole or in part[8]. Fruit wine features high nutrition, good taste and low alcohol [9].

The later the blueberry is harvested, the higher the sugar concentration mainly in the form of fructose and glucose, which could reach up to about $13 \%$. According to the U.S. Department of Agriculture, the aroma consists of aloe xylol oxide, geraniol and clove oil essence. Blueberry fruit wine, made from blueberry, mainly goes through fruit selection, crushing (sugar added), fermentation, pressing, post fermentation, filtration, bottling and other processes. Compound blueberry wine is a kind of wine made from blueberry and other materials with special functions.

\subsection{Compound fruit and blueberry wine}

In China, there are many problems in fruits processing, such as insufficient processing capacity, lack of diversity in final products and preservation as fruits are prone to rot. Compound fruit wine could be a solution to avoid waste of resources, which is a kind of fruit wine with unique flavor, balance nutrition and improved sensory quality [10].

At present, there have been numerous studies on the fermentation of blueberry wine while little attention is paid to the brewing technology of blueberry compound wine. The fermentation of blueberry compound wine is affected by many factors, such as initial $\mathrm{pH}$ value and sugar content, yeast inoculation, fermentation temperature, fermentation duration, etc. Su Junfeng [11] obtained from experiments the best formula of blueberry grape compound fruit wine, in which the ratio of fermentation juice and Cabernet Sauvignon grape fermentation juice stands at $3: 7$, with $2 \%$ of added grape fermentation juice and ethyl acetate at $600 \mu \mathrm{L} / \mathrm{L}$.

With dragon fruit and blueberry as the raw material, He Jingliang [12] studied the optimal fermentation conditions of compound fruit wine, and optimized the fermentation process of compound fruit wine through experiments, consisting of $20 \%$ initial sugar content, initial $\mathrm{pH} 3.9,1.1 \%$ of yeast inoculation while the ratio of dragon fruit and blueberry juice is set at 1:1 under the temperature of $22{ }^{\circ} \mathrm{C}$ for 9 days. Zhang Fangyan [13] explored the best brewing technology of blueberry kiwifruit compound fruit wine to find out the best 
fermentation technology conditions. He concluded that among all factors, initial sugar content is most influential on the accuracy and sensory score of fruit wine while yeast addition has the least effect on the final product. Some components of blueberry fruit are unstable in the fermentation process, such as pectin and protein, making the wine prone to browning in the brewing process and resulting in turbidity of the wine body, loss of nutrients and aroma, thus lowering the quality of the wine. In view of this situation, researchers [14] took mulberry blueberry wine as an example to study the clarification process of mulberry blueberry wine and observe the effect of gelatin, bentonite, chitosan and diatomite in different ratios on clarification, the clarification process of mulberry blueberry wine was optimized by three-level orthogonal test of four factors. The results showed that the best conditions for clarification of mulberry and blueberry wine were $0.13 \mathrm{~g} / 100 \mathrm{ml}$ gelatin, $0.09 \mathrm{~g} / 100$ $\mathrm{ml}$ bentonite, $0.08 \mathrm{~g} / 100 \mathrm{ml}$ chitosan and $0.11 \mathrm{~g} / 100$ $\mathrm{ml}$ diatomite.

\subsection{Herbal compound blueberry wine}

As people have a higher expectation for blueberry wine, herbs are widely used in the brewing process of blueberry wine to explore possibilities of diverse taste, nutrition and health function.

Tartary buckwheat has long been a kind of natural functional food, with records tracing back to ancient China, which is claimed to be effective in the prevention and treatment of tartary buckwheat, lowering blood pressure, blood sugar and blood lipid. Zhang chongjun et al. [15] took buckwheat and blueberry as the raw material to optimize the brewing process of buckwheat blueberry wine from fermentation temperature, inoculation, $\mathrm{pH}$ value, initial sugar content. And the best combination of the four indicators are determined. At the same time, the results showed that tartary buckwheat and blueberry wine are rich in antioxidant, which would increase along the wine volume.

Ginseng has a medicinal history of more than 2000 years in China [16], with a wide range of pharmacological and clinical applications. It is now widely used as a new functional food in the world. Xia Xiudong et al. [17] compared and analyzed the content of main ginsenosides in blueberry wine and blueberry blended wine added with ginseng, and concluded that ginseng can give a variety of ginsenosides to wine products, providing theoretical basis for the development of new health fruit wine.

Black garlic is a kind of healthy food made from fresh raw garlic through fermentation. Rich in polyphenol, it has a strong performance in antioxidation and anti-acidification. Ji yanru et al. [18] developed three formulas of black garlic blueberry wine, based on which the antioxidant activity, total phenol, total flavonoids and anthocyanin content were analyzed. The scavenging ability of black garlic blueberry wine to hydroxyl radicals, DPPH radicals, superoxide anion and the inhibition rate of LPO were determined, which provided a theoretical basis for the development of black garlic blueberry wine.

\section{Blueberry fruit beer}

As a beverage with low alcohol content, beer contains a variety of nutrients that are easy to be absorbed and utilized by human body, including amino acids, vitamins, inorganic salts, low molecular sugar and various enzymes. Despite a high yield of up to 44.015 million kiloliters, there is not a diverse range of beer products in China. With the growing awareness of health among Chinese consumers, they have a higher expectation for nutritional and functional beer [19]. Fruit beer is a new type of beer product with a low alcohol content and rich in tastes, thus meeting the needs of different consumers. Many fruits are used in fruit beer production, including blueberry. Blueberry beer is a kind of nutritious drink, catering people's needs for health. Compared with ordinary beer, blueberry beer has a higher level of nutrition, including protein, calcium, magnesium, potassium and vitamin as well as better flavor and greater biology and colloid stability.

The fermentation of blueberry beer is affected by many factors, with the amount of blueberry juice and yeast being the most prominent ones. The production process of blueberry beer is optimized by multiple methods. Xu Jing et al. [20] used blueberry juice and malt for fermentation at low temperature. The experiment showed that the addition of $10 \%$ blueberry juice and $0.4 \%$ yeast at the beginning of the fermentation could produce, blueberry beer superior to other processing conditions in terms of aroma and stability.

Table 1. Comparison of nutritional components between blueberry beer and general beer

\begin{tabular}{|c|c|c|}
\hline Component & Beer & Blueberry Beer \\
\hline $\begin{array}{c}\text { Carbohydrates } \\
(\mathrm{g} / \mathrm{L})\end{array}$ & 50 & 53 \\
\hline Protein $(\mathrm{g} / \mathrm{L})$ & $2-6$ & 37 \\
\hline Calcium $(\mathrm{mg} / \mathrm{L})$ & 40 & 109 \\
\hline $\begin{array}{c}\text { Magnesium } \\
(\mathrm{mg} / \mathrm{L})\end{array}$ & 107 & 20 \\
\hline \begin{tabular}{c} 
Sodium $(\mathrm{mg} / \mathrm{L})$ \\
\hline $\begin{array}{c}\text { Potassium } \\
(\mathrm{mg} / \mathrm{L})\end{array}$
\end{tabular} & 20 & 102 \\
\hline $\begin{array}{c}\text { VitaminC } \\
(\mu \mathrm{g} / \mathrm{L})\end{array}$ & 0 & $50-100$ \\
\hline $\begin{array}{c}\text { Pantothenic acid } \\
(\mu \mathrm{g} / \mathrm{L})\end{array}$ & 1682 & 1679 \\
\hline
\end{tabular}




\section{Development and utilization of blueberry wine and its pomace in food manufacturing}

Blueberry pomace is a by-product of blueberry processing that is rich in polyphenols [21], dietary cellulose [22], water-soluble antioxidant anthocyanin, and polyphenols. It is usually treated as a waste by people. Anthocyanin is the main active component in blueberry wine dregs, whose content can reach up to $71.9 \%$ in blueberry fruit, higher than that of blueberry wine [23]. Lee $\mathrm{j}$ et al. [24] reported that more than $42 \%$ of anthocyanin and more than $15 \%$ of polyphenol are stored in blueberry fruit dregs. Khanal R C et al. [25] reported that $25 \%-50 \%$ of proanthocyanidins are stored in blueberry fruit dregs. The utilization of blueberry dregs can not only protect the environment, but also produce social and economic benefits. At present, blueberry dregs have taken the place of low-precision flour in food manufacturing of jam, bread, cake and biscuit to produce high fiber products to meet the special needs of people.

Because of the health risks caused by the high sugar content in blueberry jam, researchers continue to optimize the formula and production process. Zhou Jie et al. [26] used blueberry wine dregs along with sucrose, honey, citric acid, sodium alginate and xanthan gum to make blueberry wine dregs jam. Through experiments, the best processing conditions of blueberry wine dregs jam were obtained to produce blueberry wine dregs jam with good taste, high anthocyanin content and low level of sugar.

In the production of gluten free biscuits, researchers [27] replaced the low gluten flour with blueberry fruit dregs. The optimized formula delivers a higher level of dietary fiber and mineral while there was no significant difference from products added with flour in nutritional composition, which was more beneficial to health. Rohm $\mathrm{h}$ et al. [28] reported that blueberry fruit dregs were added to bread, cake and biscuits to make low-energy, high fiber products. In addition, blueberry pomace can also be used as the ingredient of functional health food.

\section{Conclusion}

At present, most blueberry products in China by traditional processing techniques feature low utilization rate and low added value. With the growing public awareness of health and the large-scale cultivation of blueberry in China, it is particularly important to establish a systematic and scientific blueberry processing system to optimize the formula and processing techniques. The development of blueberry wine and fruit residue can not only optimize blueberry processing, but also create huge economic benefits to the industrial chain with the improving utilization rate of blueberry resources. Processing blueberries into green and healthy products with high-tech content is the key to solve the problem of blueberry development and utilization [7].

From this paper, recent studies on blueberry compound wine and fermentation technology of blueberry beer were analyzed and summarized along with proposals on the development and utilization of blueberry fruit residue in the food manufacturing industry, serving as a reference for the development and utilization of blueberry wine and the industrial processing of blueberry beer residue.

\section{Acknowledgment}

This paper was supported by Wuhan Social Science Federation under grant number WHSKL2019061.

\section{References}

1. Elks C M,Francis J,Stull A J,et al. Overview of the Health Properties of Blueberries[M]/ / Bioactives in Fruit: Health Benefits and Functional Foods. John Wiley \& Sons, Ltd,2013: 251 - 271.

2. Tsuda T. Dietary anthocyanin-rich plants: biochemicalbasis and recent progress in health benefits studies [J]. Mol. Nutr. Food Re, 2012, 56(1):159-170.

3. Grace M H, Ribnicky D M, Kuhn P, et al. Anthocyanins extracted from Chinese blueberry (Vaccinium uliginosum L) and its anticancer effects on DLD-1 and COLO205 cell [J]. Chin Med,2010, 123(19):2714-2719.

4. Kader F,Rovel B. Fractionation and klentification of the phenolic compounds of highbush (Vaccinium Corymbosum L) [J] .Food Chem,1996,55(1):3540.

5. Guyi,Heshanan. Blueberry and cranberry [M] .Beijing:China Agriculture Press,2001.

6. Liyadong.High quality and high yield cultivation techniques of Blueberr [M] .Beijing:China Three Gorges press,2007:2.

7. Wangzhitong.Study on the new processing technology of blueberry wine[D]. Changcun:Jilin Agricultural University, 2007.

8. Daiguizhi.On the current situation and development strategy of fruit wine industry in China[J]. Storage and Process, 2002, 2(6): 2-5.

9. Liguohong, Zhangdahe, Panjianjun. Using local resources to develop low nutrition fermented wine[J]. Liquor making technology,2003(5):90 93.

10. Lizhenping. Chinese wine culture[M]. Beijing: Current Affairs Publishing House,2007.

11. Su Junfeng.Optimization of blueberry grape wine by orthogonal design[J]. Northern gardening,2010(6):188-191.

12. He Jingliang.Study on fermentation technology of dragon fruit blueberry compound fruit wine[J]. Journal of Heze University,2017,39(5):86-90.

13. Zhang Fangyan.Study on the brewing technology of blueberry kiwifruit compound wine $[\mathrm{J}]$. Liquor making technology,2016(12):87-90. 
14. Ke Quqing.Study on response surface optimization of clarification technology of mulberry and blueberry wine with compound clarifier[J].Food industry,2018,39(1):89-91.

15. ZhangChongjun.Study on the production technology and antioxidation of tartary buckwheat and blueberry wine[J].Food industry,2017,38(9):90-92.

16. Fengyan.Research progress in pharmacological action and clinical application of ginseng[J].Clinical research of traditional Chinese Medicine,2013(6) : $121-122$.

17. Xia Xiudong.Determination of main ginsenosides in blueberry ginseng health wine[J].Journal of Jiangxi agriculture,2017,29(11):91-94.

18. Ji Yanru.Study on antioxidant capacity and active components of black garlic blueberry wine[J]. Journal of food science and technology,2017,35(2):65-69.

19. Shi jinglue.Development of blueberry beer[J]. China brewing.2009(1): 175-177.

20. $\mathrm{Xu}$ Jing.Study on the production technology of blueberry beer[J].Liquor Making.2009,35(5):74-76.

21. KHANAL R C, HOWARD L R, WILKES S E, et al. Effect of dietary blueberry pomace on selected metabolic factors associated with high fructose feeding in growing sprague-dawley rats $[\mathrm{J}]$. J Med Food, 2012,15(9): 802-810.

22. HE B, ZHANG L L, YUE X Y, et al. Optimization of ultrasound-assisted extraction of phenolic

23. Compoundsand anthocyanins from blueberry Vaccinium ashei wine pomace[J]. Food Chem, 2016, 204: 70-76.

24. LEE J, DURST R W, WROLSTAD R E. Impact of juice processing on blueberry anthocyanins and polyphenolics: comparison of two pretreatments [J]. J Food Sci, 2010, 67(5): 1660-1667.

25. KHANAL R C, HOWARD L R, PRIOR R L. Effect of heating on the stability of grape and blueberry pomace procyanidins and total anthocyanins[J]. Food Res Int, 2010, 43(5): 1464-1469.

26. ZhouJie.Optimization of the processing technology of blueberry dregs jam[J].China brewing.2018, 37(4): 201-204.

27. SARICB, MISANA, MANDICA, et al. Valorisation of raspberry and blueberry pomace through the formulation of value-added gluten-free cookies[J]. J Food Sci Technol, 2016, 53(2): 1140-1150.

28. ROHM H, BRENNAN C, TURNER C, et al. Adding value to fruit processing waste: innovative ways to incorporate fibers from berry pomace in baked and extruded cereal-based foods-a susfood project[J]. Foods,2015, 4(4): 690-697. 